\title{
Origin and behaviour of emphysematous bullae
}

\author{
M D L MORGAN," C W EDWARDS, J MORRIS, H R MATTHEWS \\ From the Departments of Respiratory Medicine, Histopathology, and Thoracic Surgery, East Birmingham \\ Hospital, Birmingham
}

ABSTRACT Giant emphysematous bullae are believed to produce symptoms of pulmonary compression and collapse by containing gas under pressure that has been generated through valvular feeding airways. To examine this hypothesis, we have measured oxygen and carbon dioxide tensions $\left(\mathrm{Po}_{2}, \mathrm{PCO}_{2}\right)$ in four patients and pressure within the bullae in three patients immediately before surgery. During spontaneous tidal respiration $\mathrm{Po}_{2}$ in the bulla was higher than arterial $\mathrm{Po}_{2}$ but did not rise as fast during the breathing of oxygen. The intra-bulla pressure during inspiration was negative $\left(-5.5\right.$ to $\left.-19 \mathrm{~cm}_{2} \mathrm{O}\right)$ and similar to pleural pressure in phase and degree. During intermittent positive pressure ventilation in two patients airway pressures were transmitted to the bulla with the development of a positive end expiratory pressure within the bulla. Histological examination of the walls of the bullae in the four patients and adjacent lung tissue in an additional patient failed to identify any valvular mechanism. The available information suggests that bullae develop after retraction and collapse of surrounding lung away from a region of weakness.

\section{Introduction}

Surgical removal of a large bulla offers a few patients with chronic lung disease the chance of a dramatic improvement in their condition. The definition of bullous emphysema in life has been much improved in recent years with the development of computed tomography. ${ }^{12}$ Consequently, knowledge of the size and position of a bulla now helps in the decision to remove it. ${ }^{34}$ It has also been shown recently that successful surgery does not increase the rate of decline of background lung disease or encourage the growth of new bullae. ${ }^{5}$ Despite this new knowledge, little attention has been paid to the investigation of mechanisms through which bullae might arise and cause symptoms, and why any improvement should be expected after surgery.

The most commonly stated view of bulla formation is that a weak area of lung is fed by an airway that behaves like a one way valve, allowing gas to enter the region but impeding its exit. Such a space is thought to grow because it is preferentially inflated during spontaneous respiration or enlarged through coughing. The effect of such a bulla, believed to contain gas under pressure, would be to compress and collapse the surrounding lung. The justification for surgery there-

Address for correspondence: Dr M D L Morgan, Glenfield General Hospital, Leicester LE3 9QP. (Reprints will not be available.)

Accepted 10 April 1989 fore would arise from the identification of compressed lung and improvement obtained from its re-expansion.

On examination such a theory, though widely held, seems improbable as it requires several coincidences that are not supported by the available facts. In particular, when measurements of pressure have been made within bullae they have not confirmed the existence of compression, and have even suggested the opposite. To investigate this and other contradictions, we have made some direct measurements of gas content and pressure within bullae and have examined the histological appearances of the lung surrounding bullae. Our findings and a review of the published reports suggest an alternative explanation for the evolution of emphysematous bullae and a more rational explanation for the success of surgery.

\section{Methods}

\section{PATIENTS}

We studied four men (aged 41-64 years) who were undergoing surgery for removal of giant emphysematous bullae (table). The decision to operate had been made on the grounds of symptoms, radiological appearance, and surgical accessibility of the bulla. Spirometry in all patients showed expiratory airflow limitation (FEV, $21-69 \%$ predicted), hyperinflation (total lung capacity $106-134 \%$ predicted), poor carbon monoxide gas transfer when corrected for alveolar volume (KCo-49-69\% predicted), and a 
Details of the patients

\begin{tabular}{lllllll}
\hline $\begin{array}{l}\text { Patient } \\
\text { No }\end{array}$ & $\begin{array}{l}\text { Age } \\
(y)\end{array}$ & $\begin{array}{l}\mathrm{FEV}_{1} \\
l(\% \mathrm{O})\end{array}$ & $\begin{array}{l}\mathrm{PaO}_{2} \\
(\mathrm{KPa})\end{array}$ & $\begin{array}{l}\mathrm{PaCO}_{2} \\
(\mathrm{kPa})\end{array}$ & $\begin{array}{l}\mathrm{PbO}_{2} \\
(\mathrm{kPa})\end{array}$ & $\begin{array}{l}\mathrm{PbCO}_{2} \\
(\mathrm{KPa})\end{array}$ \\
\hline 1 & 59 & $1.91(67)$ & 9.6 & 4.4 & 13.5 & 3.6 \\
2 & 52 & $0.90(22)$ & 6.3 & 5.6 & 13.3 & 5.0 \\
3 & 64 & $0.71(24)$ & 9.0 & 5.1 & 11.9 & 4.9 \\
4 & 41 & $1.37(34)$ & 8.7 & 6.0 & 12.8 & 5.6 \\
\hline
\end{tabular}

FEV 1 - forced expiratory volume in one second; $\% \mathrm{P}$-percentage of predicted value; $\mathrm{PaO}_{2}, \mathrm{PaCO}_{2}$ - arterial oxygen and carbon dioxide tensions; $\mathrm{PbO}_{2}, \mathrm{PbCO}_{2}$ - bulla oxygen and carbon dioxide tensions.

Patient 1-History of dyspnoea for five years, exercise tolerance about $18 \mathrm{~m}$. Computed tomography showed a large, multilocular bulla arising from the right upper lobe and extending anteriorly to the lower chest.

Patient 2-Very large bulla in the right chest. Computed tomography showed no lung but a small remnant did expand to fill the whole chest after surgery; some emphysema in the left upper lobe.

Patient 3-Computed tomography showed a large bulla in the right lower lobe with posterior extension behind the right upper lobe and collapse of the right lung against the paravertebral region.

Patient 4-Recurrent pneumothorax and increasing dyspnoea for two years. Bulla in the right lower lobe not very clear on plain chest radiograph but easily visible on the computed tomogram.

degree of hypoxaemia (arterial $\mathrm{Po}_{2} 6 \cdot 2-11.9 \mathrm{kPa}$ ). In each case the procedure consisted of a full thoracotomy with excision of the dome of the bulla and plication of the base of the bulla (where it joins normal lung) with strips of Teflon tape. ${ }^{6}$ After giving full informed consent and receiving standard premedication, the patients were settled supine on the operating table. Before induction of anaesthesia a fine (21 gauge) needle was inserted percutaneously into the bulla with local anaesthesia. The needle was guided by the preoperative computed tomogram and its position was later verified at thoracotomy. The needle was used subsequently to withdraw gas samples and record pressure within the bulla during spontaneous respiration and during intermittent positive pressure ventilation after induction.

\section{GAS SAMPLES}

Gas content in the bulla was examined at one minute intervals during spontaneous tidal respiration for six minutes. After two minutes the patient breathed an oxygen rich mixture $(7 \mathrm{l} / \mathrm{min}$ via a Magill circuit and facemask) so that the wash in of oxygen could be observed. The gas samples were withdrawn in $10 \mathrm{ml}$ aliquots into a glass syringe and analysed immediately

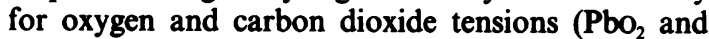
$\mathrm{PbCO}_{2}$; Corning 170 gas analyser). Arterial blood gas tensions were analysed from simultaneous samples of arterial blood taken from an indwelling radial arterial cannula (previously inserted for anaesthetic monitoring) and transported on ice. For later comparison the ideal alveolar oxygen tension $\left(\mathrm{PAO}_{2}\right)$ was calculated from the full aveolar gas equation.'

\section{INTRA-BULLA PRESSURE}

Measurement of intra-bulla pressure was made directly in three patients, and compared with pleural pressure. To achieve this, the patients had previously swallowed a $10 \mathrm{~cm}$ latex oesophageal balloon contain-
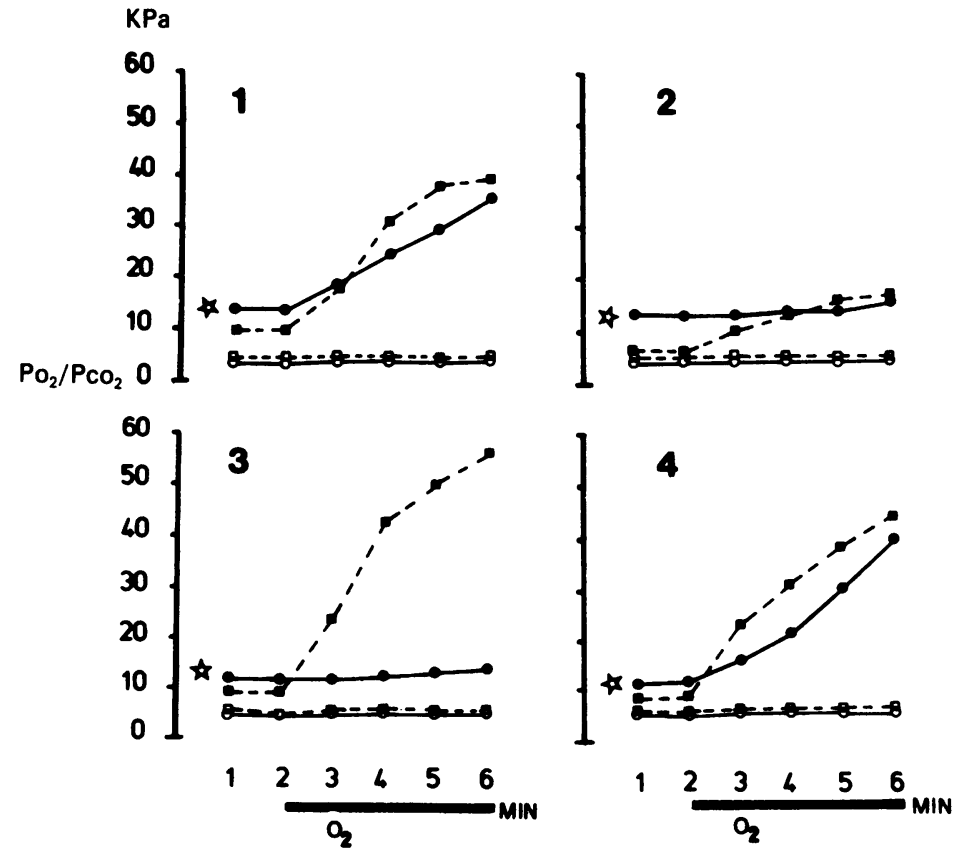

Fig 1 Samples of bulla gas and arterial blood gas tensions in four patients taken while they were breathing room air for two minutes and then facemask oxygen for four minutes. Initially bulla oxygen tension $\left(\mathrm{PO}_{2}\right)(\mathrm{O}-\mathrm{O})$ is always greater than arterial $\mathrm{PO}_{2}\left(\mathrm{Q}_{-}-\mathrm{U}\right)$ and similar to calculated alveolar $\mathrm{PO}_{2}$ (4) during the breathing of air, but it does not rise as rapidly with oxygenation. In patients 2 and 3 , the rate of rise of bulla $\mathrm{PO}_{2}$ is slower, indicating reduced ventilation, while patient 2 also shows a poor response of arterial $\mathrm{PO}_{2}$, suggesting an additional physiological shunt. Bulla $\mathrm{PCO}_{2}(\mathrm{O}-\mathrm{O})$ is lower than arterial $\mathrm{PCO}_{2}(\square--\square)$ and remains so. 


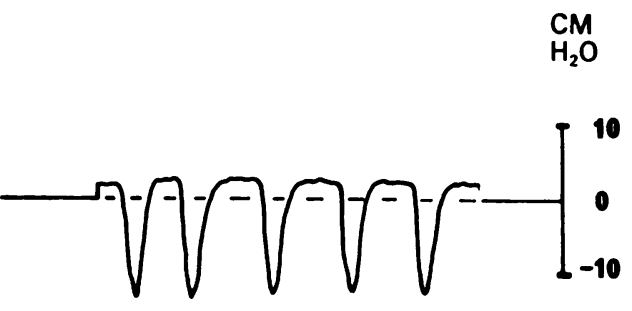

$\uparrow$

needle in bulla

Fig 2 Pressure within the bulla immediately after insertion of the needle into the bulla in patient 2. The record shows negative pressure during tidal inspiration. Removal of gas for sampling made no detectable difference to the absolute pressure record.

ing $0.5 \mathrm{ml}$ air. The needle and the balloon were connected to identical catheter-transducer systems (Druck Ltd), referenced to atmospheric pressure, and attached to paper recorders. The frequency responses characteristic of the two systems were similar $(90 \%$ response to square wave signal $=150 \mathrm{~ms}$ ). The pressures within the bullae were recorded continuously from the moment of insertion of the needle until the induction of anaesthesia. In two cases the measurement was continued for a few minutes during intermittent positive pressure ventilation until the bullous lung was deflated. In these cases the measurement of airway pressure was added to the record.

\section{HISTOLOGY}

Modern surgical techniques conserve as much functioning lung tissue as possible, and in our four cases

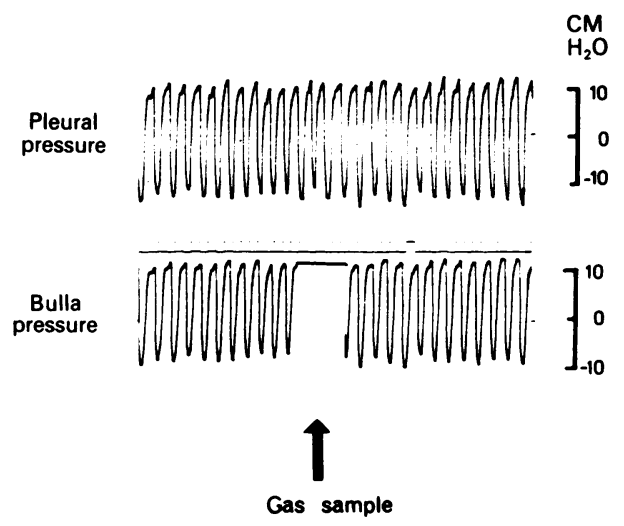

Fig 3 Simultaneous record of pleural and bulla pressure in patient 3, showing that the two are in phase, though the amplitude of the bulla changes are about $10 \%$ less than that of the pleural changes.

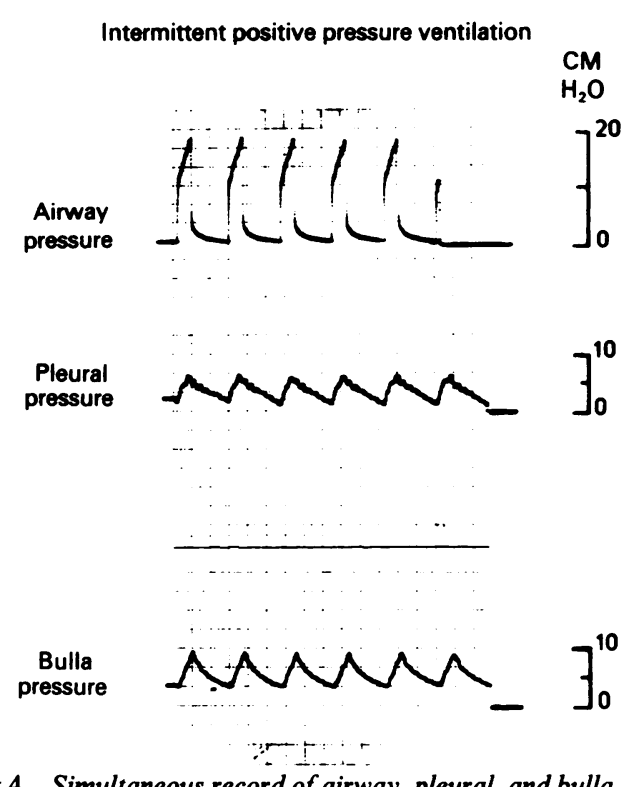

Fig 4 Simultaneous record of airway, pleural, and bulla pressure during intermittent positive pressure ventilation in patient 4, showing that the airway pressure is transmitted to the bulla but is attenuated. A positive end expiratory pressure of $5 \mathrm{~cm} \mathrm{H}_{2} \mathrm{O}$ develops in the bulla.

only the walls of the bullae were available for examination by light microscopy and electron microscopy; but we were also able to examine material from a fifth patient, who had required a lobectomy in which a lobe containing a bulla was removed. The junctional region between bulla and normal lung in this specimen was examined closely. In all cases the material to be examined by light microscopy was embedded in paraffin wax and sections were treated with haematoxylin and eosin and elastic van Gieson stains.

Blocks were taken from the formalin fixed specimens for electron microscopic examination and sections were stained with uranyl acetate and lead citrate by a conventional technique.

\section{Results}

\section{GAS CONTENT}

The records of $\mathrm{PbO}_{2}$ and $\mathrm{PbCO}_{2}$ and of $\mathrm{PaO}_{2}$ and $\mathrm{PaCO}_{2}$ for all four patients are illustrated in figure 1. The samples at one minute and two minutes represent gas taken during tidal respiration with the patient breathing room air and the remaining four samples while they were breathing oxygen. In each case the initial $\mathrm{PO}_{2}$ in the bulla was higher than arterial $\mathrm{Po}_{2}$ and was virtually identical to the calculated alveolar $\mathrm{PO}_{2} . \mathrm{PCO}_{2}$ within the bulla was lower than arterial $\mathrm{PCO}_{2}$. With oxygenation there was a rise in bulla $\mathrm{Po}_{2}$, but this was 
Morgan, Edwards, Morris, Matthew 청
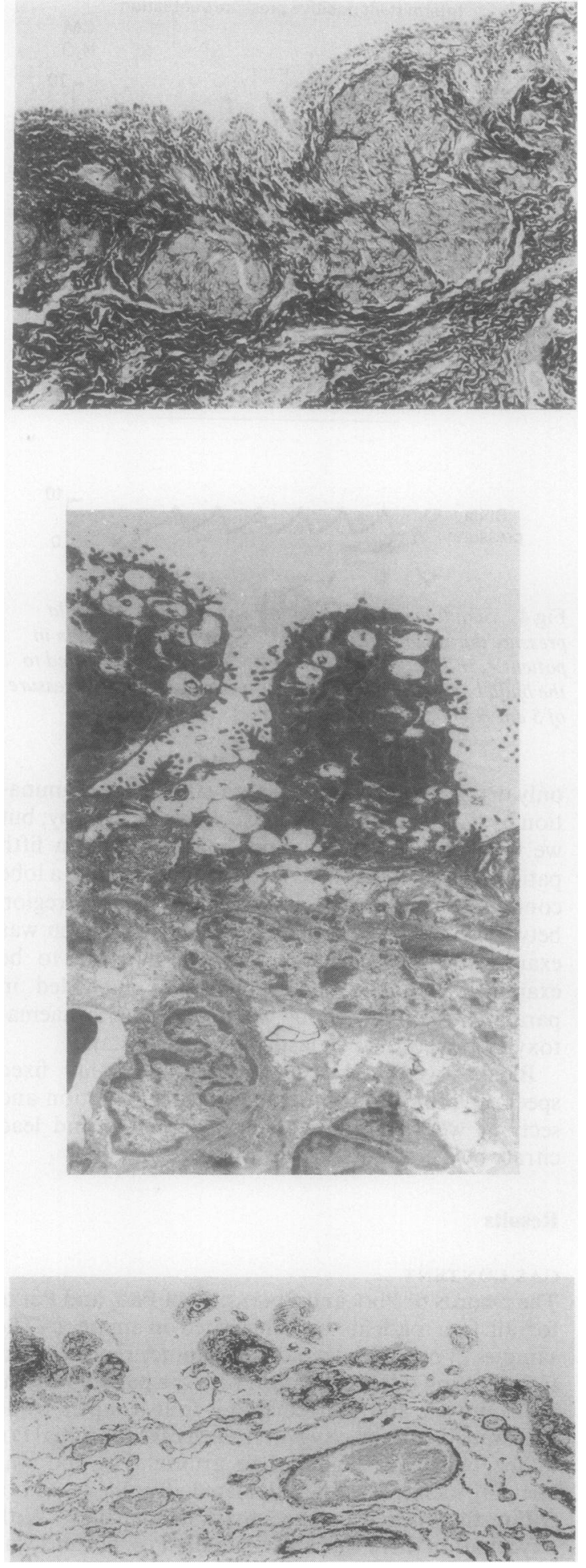

always exceeded by the rate of rise of arterial $\mathrm{Po}_{2} . \stackrel{\times}{.}$ There were some individual differences: patients 1 and 4 showed a more rapid rise in $\mathrm{PbO}_{2}$, and in patient 20 $\mathrm{PaO}_{2}$ did not rise as rapidly as in the others. $\mathrm{PbCO}_{2}$ dido not change in any patient. No patient reached $\overline{0}$. equilibrium within the four minutes of oxygen wash in. $\mathbb{\Phi}$

\section{PRESSURES}

Intrabullar pressure was recorded from the moment of $\vec{\circ}$ insertion (fig 2). The mean bulla pressure was always negative. Peak inspiratory pressure ranged from $-5 \cdot 5 \mathrm{\omega}$ to $-19 \mathrm{~cm} \mathrm{H}_{2} \mathrm{O}$ and end tidal expiratory pressures from $+2 \cdot 5$ to $+11 \mathrm{~cm} \mathrm{H}_{2} \mathrm{O}$. Bulla pressure followed pleural pressure almost exactly (fig 3 ). The phase was ${ }^{+}$ identical but the amplitude of the pressure change in the bulla was about $10 \%$ less than the amplitude of the pleural pressure swing. Removal of gas for samplingo made no detectable difference to the absolute pressure record.

In two patients the measurements were continued during intubation and intermittent positive pressure $\vec{c}$ ventilation. In an example from one patient (fig 4) the 0 airway pressure was transmitted to the bulla but was. attenuated by about $75 \%$. In this case the amplitude of the bulla pressure excursions were slightly greater than the amplitude of the oesophageal pressure changes. The most obvious feature was the development of ano equilibrated end expiratory pressure of $5 \mathrm{~cm} \mathrm{H}_{2} \mathrm{O}$, which was not present in the airway.

\section{HISTOLOGY}

The walls of the bullae were up to $2 \mathrm{~mm}$ thick and? consisted of fibroelastic and muscular tissue of unknown origin, containing arterial vessels with $a$ 응 prominent muscular media (fig $5 a$ ). The bullae werex lined by cuboidal, non-ciliated cells, which on ultra- $\frac{-}{3}$ structural examination showed the characteristic features of granular pneumocytes (fig $5 b$ ). Examina-o tion of the lobectomy specimen showed that the floor of the bulla consisted of alveolar ducts and alveolio opening directly into the lumen (fig $5 c$ ). There was some interstitial fibrosis and proliferation of smooth muscle adjacent to the lumen but no valvular mechan ism was seen.

Fig 5 (a) Wall of an emphysematous bulla lined by cuboidal cells. Note the muscular thick walled vessels within the walls and the abundant elastic fibres. (Elastic-Van Gieson.) (b) Electron micrograph showing the lining cells of a bulla to have the characteristics of type II cells, with microvilli and intracytoplasmic lamellated bodies. (Uranyl acetate and lead citrate.) (c) Junction between the bulla and normal lung; alveoli open directly into the lumen and their walls are thickened at this point. (Elastic-Van Gieson.) 
Discussion

The conventional view of the evolution of bullae is that a disease process, usually emphysema, produces a localised area of parenchymal destruction supplied by airways that contain a valvular obstruction, allowing gas to enter but not to leave the region. Continued alveolar destruction and the inflating effect of gas entering the space blow it up, and the resultant expansion of the bulla causes compression and collapse of the surrounding lung. Surgical treatment is aimed at relieving the pressure and allowing the lung to re-expand. The demonstration of compressed lung is therefore considered a prerequisite of improvement after surgery.

Although several features of this theory are inconsistent and contrary evidence has been available for many years, the theory has never been seriously challenged. There must be local defects to begin the process and these could be regions of traumatic damage or emphysema that coalesce. A bulla is most unlikely, however, to grow in the manner that has been described. The origin of this explanation comes from the histological observations of Hayashi ${ }^{8}$ in 1914, cited by Head and Avery. ${ }^{9}$ Surgeons can now observe the floor of a bulla directly when it is partly inflated, and see openings that are widely patent. In the lobectomy specimen in this study the lung parenchyma communicated directly with the lumen of the bulla. No evidence of a valvular mechanism was apparent. Of course, these openings could possibly be floppy and close prematurely at low transpulmonary pressures, like the surrounding emphysematous lung. It is difficult, however, to see how such a mechanism could maintain positive pressure in a bulla and even more difficult to understand how the pressure was generated in the first place. A bicycle pump mechanism implies either that extreme negative pleural pressures result in preferential inflation of the bulla or that the bulla is filled in expiration or during coughing. The latter is unlikely as expiratory positive pleural pressure will be applied equally to the bulla and the lung and the gas should follow the pressure gradient to the mouth. Conceivably pressures transmitted through the walls of bullae during coughing could enlarge an area of weakness but this pressure would not be maintained during normal respiration.

Direct observation of the volume change and ventilation of bullae by computed tomography also contradicts the conventional view. ${ }^{4} \mathrm{~A}$ bulla observed in inspiration and expiration does not change size to any appreciable degree and the change that does occur is always in phase (that is, growth during inspiration and empyting during expiration). Furthermore, our measurements show that under tidal conditions the bullae contain gas that is identical to that in alveoli.
When the inspired oxygen is increased $\mathrm{Po}_{2}$ rises more slowly in the bulla than in the arterial blood, indicating that, although ventilation occurs, it is slower than the surrounding lung. How ventilation occurs is not known. Gas could enter the bulla through a residual feeding airway or collaterally via adjacent alveoli. Overall gas flow into and out of bullae appears to be small but unimpeded and the bullae rarely contribute to excessive deadspace ventilation. ${ }^{1011}$

The evidence that a bulla compresses surrounding lung is based on observation of adjacent lung that appears collapsed on plain chest radiographs and computed tomograms or at operation. Often such collapsed lung expands during surgery and may be responsible for subsequent improvement in function. It is indisputable that collapsed lung may adjoin a bulla but the lung need not have been compressed for this to occur. In fact, computed tomography often shows no evidence of adjacent collapse and the computed tomographic density of peribullous lung is not increased by compression. Some confirmation of compression would be obtained if the pressure within the bulla were shown to be higher than the pleural pressure acting on the surrounding lung. Although a direct measurement of intra-bulla pressure made many years ago recorded a pressure of $-11 \mathrm{~cm} \mathrm{H}_{2} \mathrm{O}$ during tidal breathing and $-27 \mathrm{~cm} \mathrm{H}_{2} \mathrm{O}$ during a maximal inspiration, ${ }^{12}$ most writers still describe such bullae as "tension" cysts that exert compression on surrounding lung.

In this study we have measured pressures within the bulla and related them to airway and pleural pressures measured simultaneously. The use of oesophageal balloon measurements to record pleural pressure has been shown to be valid, giving results that are accurate to within $10 \%$ and independent of lung volume and posture. ${ }^{1314}$ Our estimated pleural pressure swings are about $10 \%$ greater than the bulla signal, but the limitations of the method are such that little importance can be attached to this finding. Nevertheless, the bulla pressures were never positive in inspiration and never more positive than pleural pressure at end expiration, and the pressure swings were always in phase. This would suggest that at least during spontaneous respiration bullae are spaces that are subject to the same forces as the surrounding lung and respond in a similar manner.

During positive pressure ventilation, with the chest open or closed, the circumstances are different and bullae are exposed directly to filling pressures that produce local positive end expiratory pressure within them. This may enlarge the bulla rapidly during the exposure and make it vulnerable to rupture. Coughing during spontaneous ventilation does not seem to have the same effect. We have coincidentally observed the rise in pressure during a cough, which appears as great 
but also as transient as the change in pleural pressure. The cough impulse is therefore transmitted directly to the bulla by compression rather than via the airway. It is possible, however, that coughing contributes to growth of a bulla not by inflating it but by compressing it and tearing its edge.

If a bulla is not blown up, how then does it inflate preferentially and take up the spherical shape that it often has? This is answered partly by the observations of Ting, who examined the pressure-volume characteristics of isolated bullae and the resected specimens of adjacent lung. ${ }^{15} \mathrm{He}$ found that bullae behave like paper bags, which are extremely compliant until they are full, when they become tense. The lung surrounding the bulla is less compliant and the pressure required to inflate it exceeded the pressure necessary to inflate the bulla. This means that when bulla and lung are exposed to the same negative intrapleural pressure the bulla will fill preferentially and always be completely full before the lung.

This information now provides a framework for a rational explanation of bulla formation and the mechanism by which symptoms are produced. Once a parenchymal weakness in the lung exceeds a certain size it will result in a space within the lung that fills preferentially. The force of elastic recoil will produce retraction of the surrounding lung away from the hole and enlarge it. Even though the bulla remains in free communication with the airway it will not participate in ventilation to any important extent because of its large volume, or to gas exchange because of its relatively reduced and avascular internal surface area. We need no longer invoke compression of surrounding lung as a cause of pulmonary collapse because the lung has simply shrunk away from the space, and the resultant loss of elastic recoil on adjoning airways may well lead to occlusion and atelectasis. In support of this, it has already been shown that airway obstruction in non-smoking patients with bullae can be accounted for entirely by the loss of elastic recoil. ${ }^{16}$ Furthermore, the efforts that the chest wall would make to inflate lung that is partially collapsed will lead to both hyperinflation and the disproportionate sense of dyspnoea characteristic of these patients.

These observations also make the effects of surgery easier to explain. The effect of bullectomy and plication is not so much to ablate a space as to reconstruct the parenchyma of the lung in the manner of darning a stocking, restoring its architecture and mechanical linkage with the chest wall, while allowing deflated lung to regain its elastic properties.
Grateful thanks are due to consultant anaesthetists $\mathrm{Dr}$ R B Hopkinson and Dr M C Clapham for their cooperation.

\section{References}

1 Fiore D, Biondetti PR, Sartori F, Calabro F. The role of computed tomography in the evaluation of bullous lung disease. J Comput Assist Tomogr 1982;6:105-8.

2 Morgan MDL, Strickland BS. Computed tomography in the assessment of bullous lung disease. Br J Dis Chest 1984;78:10-25.

3 Carr DH, Pride NB. Computed tomography in preoperative assessment of bullous emphysema. Clin Radiol 1984;35:43-5.

4 Morgan MDL, Denison DM, Strickland BS. The value of computed tomography in the assessment of bullous lung disease. Thorax 1986;41:855-62.

5 Pearson MG, Ogilvie C. Surgical treatment of emphysematous bullae: late outcome. Thorax 1983;38: 134-7.

6 Parmar JH, Hubbard WG, Matthews HRW. Teflon strip pneumostasis for excision of giant emphysematous bullae. Thorax 1987;42:144-8.

7 Cotes JE. Lung function: principles and application in medicine. 4th ed. Oxford: Blackwell, 1978.

8 Hayashi J. Ueber totlichen Pneumothorax durch Infarkt und Emphysema. Frankfurt Ztschr Pathol 1914;16: $1-36$.

9 Head JR, Avery EE. Intracavitary suction (Monaldi) in the treatment of emphysematous bullae and blebs. $J$ Thorac Cardiovasc Surg 1949;18:761-76.

10 Potgeiter PD, Benatar SR, Hewitson RP, Ferguson AD Surgical treatment of bullous lung disease. Thorax 1981;36:885-90.

11 Pride NB, Hugh-Jones P, O'Brien EN, Smith LA. Changes in lung function following the surgical treatment of bullous emphysema. $Q J$ Med 1970;153:49-69.

12 Kaltreider NL, Fray WW. Pathological physiology of pulmonary cysts and bullae. Am J Med Sci 1939;197: 62-77.

13 Behrakis PK, Baydur A, Jaeger MJ, Milic-Emili J. Lung mechanics in sitting and horizontal positions. Chest 1983;4:643-6.

14 Baydur A, Cha EJ, Sassoon CH. Validation of oeso- O phageal balloon technique at different lung volumes. J Appl Physiol 1987;62:315-21.

15 Ting EY, Klopstock R, Lyons HA. Mechanical properties of pulmonary cysts and bullae. Am Rev Respir Dis 1963;87:538-44.

16 Gelb AF, Gold WM, Nadel JA. Mechanisms limiting airflow in bullous lung disease. Am Rev Respir Dis : 1973;107:571-8. 